\title{
Acquired dysfibrinogenaemia masquerading as disseminated intravascular coagulation in acute pancreatitis
}

\author{
J T WILDE, * W E G THOMAS, $\dagger$ D A LANE, $\ddagger$ M GREAVES, ${ }^{*}$ F E PRESTON*
}

From the University Departments of *Haematology, $\uparrow$ Surgery, the Royal Hallamshire Hospital, Sheffield, and the $\ddagger$ Department of Haematology, Charing Cross Hospital, London

SUMMARY Acquired dysfibrinogenaemia as the cause of coagulation abnormalities occurred in a case of acute pancreatitis. Initial coagulation studies showed a prolonged thrombin time and increased concentrations of serum fibrinogen/fibrin degradation products and plasma D-dimer. Further studies on purified fibrinogen showed evidence of degradation of the C-terminal ends of the A-alpha chains, which, it is suggested, resulted from the action of circulating pancreatic proteases. Fibrin polymerisation was thus shown to be impaired, which explains the prolongation of the thrombin time. There was a temporal relation between increased amylase activities and the prolonged thrombin time, both of which returned to normal three weeks after admission. Acquired dysfibrinogenaemia may be an underrecognised phenomenon in acute pancreatitis and may lead to misinterpretation of coagulation test abnormalities.

Disseminated intravascular coagulation (DIC) with accompanying activation of the fibrinolytic system is a recognised complication of acute pancreatitis. ${ }^{1} \mathrm{We}$ report the association of acute pancreatitis with a coagulation abnormality caused in part by probable mild DIC but predominantly by a previously undescribed acquired abnormality of figrinogen. Failure to recognise this acquired dysfibrinogenaemia may lead to a misinterpretation of coagulation test data.

\section{Case report}

A 45 year old woman presented with a two month history of epigastric pain radiating through to her back. She had lost 2 stone in weight over the previous six months but admitted to dieting. Examination showed that she was thin and anxious, with a tender epigastric mass. There was no hepatosplenomegaly or lymphadenopathy.

Her haemoglobin concentration was $8 \cdot 2 \mathrm{~g} / \mathrm{dl}$ (red cell changes consistent with iron deficiency); white cells were $9.7 \times 10^{9} / 1$, normal differential; platelet count was $558 \times 10^{9} / 1$; erythrocyte sedimentation rate was $61 \mathrm{~mm} /$ first hour; urea, electrolytes, and liver

Accepted for publication 26 January 1988 function tests were all normal. Amylase activity was $3950 \mathrm{U} / 1(70-300)$.

Prothrombin time was 16 seconds (control 15), activated partial thromboplastin time 41 seconds (43), thrombin time 32 seconds (12), reptilase time 19 (16), fibrinogen (Clauss) $2 \cdot 1 \mathrm{~g} / \mathrm{l}$ (normal range 1.6-3.9 g/l), Wellcotest serum fibrinogen degradation products, were $256 \mu \mathrm{g} / \mathrm{ml}$ (normal range $<8$ ), MabCo Dimertest plasma D-dimer $800 \mathrm{ng} / \mathrm{ml}$ (normal range <200), MabCo ELISA plasma D-dimer $2000 \mathrm{ng} / \mathrm{ml}$ (normal range $<200$ ). The prolonged thrombin time was unaffected by the addition of protamine sulphate.

\section{Pathology}

An abdominal ultrasound scan identified a mass in the epigastrium closely associated with the pancreas. A computed axial tomography scan of the upper abdomen showed a large cystic mass in the tail of the pancreas and a similar smaller lesion in the head of the pancreas. Needle aspiration of the larger mass showed a sparsely cellular aspirate containing some neutrophils and foamy macrophages. These appearances were consistent with aspirate from a pancreatic cyst. No malignant cells were seen. Episodic acute pancreatitis with cyst formation was diagnosed.

Endoscopic retrograde cholecystopancreatography showed that the pancreatic and common bile ducts 


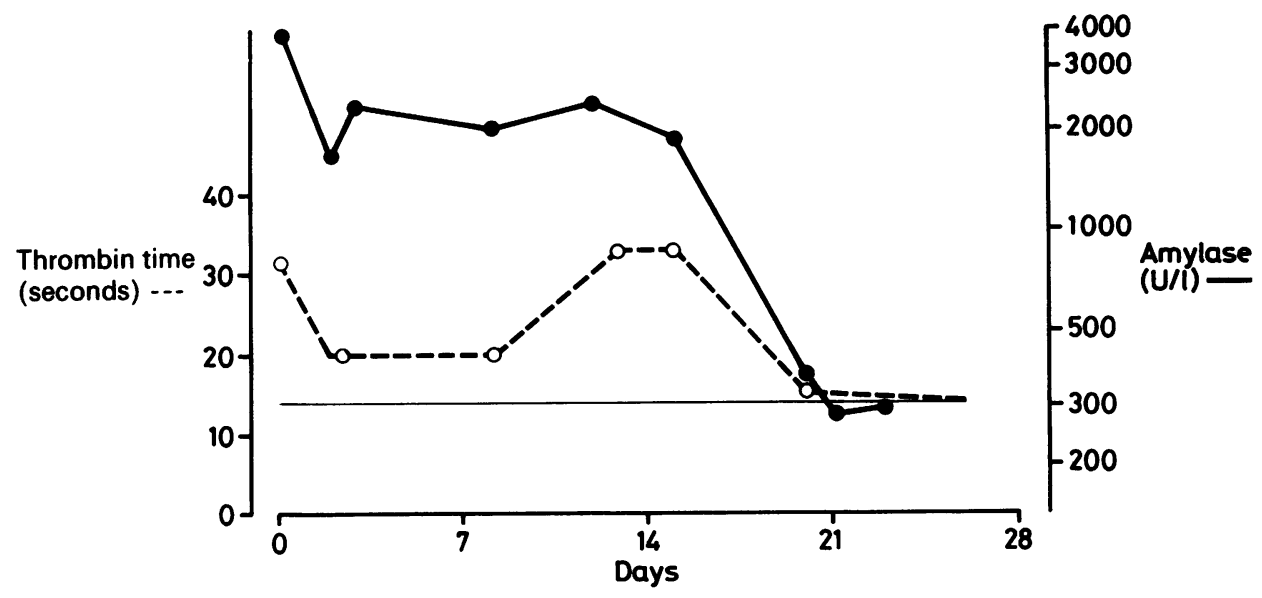

Fig 1 Temporal relation between prolonged thrombin time and raised amylase activity during first three weeks of admission. (Solid horizontal line indicates upper limit of normal for both variables).

were full of stones. These were subsequently removed at laparotomy. The patient made an uneventful recovery and returned home. Her thrombin time

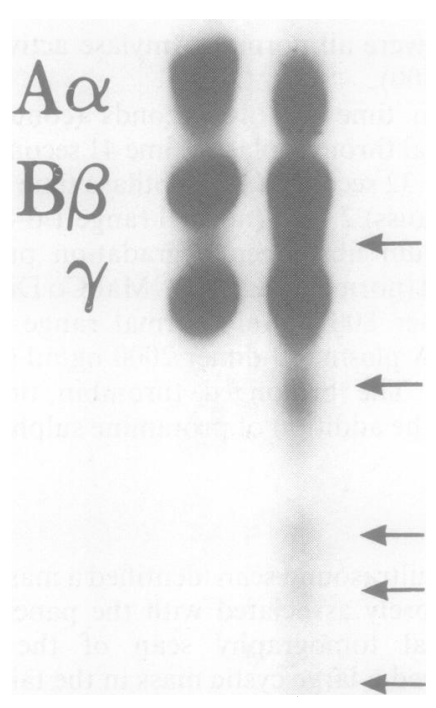

Fig 2 SDS-polyacrylamide gel electrophoresis of individual chains of reduced fibrinogen stained with Coomassie blue. Normal control fibrinogen is shown on the left, patient's fibrinogen on the right. Arrows indicate cleavage fragments resulting from A-alpha chain degradation. remained prolonged during the first three weeks of her admission; fig 1 shows the relation between this and amylase activity during this period.

The following tests were performed subsequently to investigate the cause of the prolonged thrombin time.

\section{ANALYSIS OF FIBRINOPEPTIDE RELEASE FROM FIBRINOGEN}

Analysis by high performance liquid chromatography (HPLC) of the release of fibrinopeptides A and B by the action of thrombin on the patient's fibrinogen in plasma was carried out, as described by Southan et al. ${ }^{2}$ There was a normal elution profile of fibrinopeptides but the fibrinopeptide A to fibrinopeptide B ratio of $0 \cdot 88: 1$ was reduced (theoretical value $1: 1$ ).

\section{PURIFICATION AND STRUCTURAL PROBING OF FIBRINOGEN}

Fibrinogen was purified from the patient's plasma by repeated precipitation with $\beta$-alanine. ${ }^{3}$ Analysis of this fibrinogen by HPLC showed a fibrinopeptide A to B ratio of $0.97: 1$, essentially identical with that of a fibrinogen sample obtained from a normal control subject processed simultaneously. This indicated that any fibrin monomer present in the patient's plasma had been removed during the purification procedure and that the fibrinogen was intact at its $\mathrm{N}$-terminus.

Fig 2 shows that sodium dodecyl sulphate polyacrylamide gel electrophoresis ${ }^{4}$ of the patient's fibrinogen, performed under reducing conditions using $\beta$ mercaptoethanol, was more heterogeneous than the control sample. The patient's A-alpha chains migrated more rapidly than the normal control Aalpha chains. There was also clear evidence of the presence of A-alpha chain remnants of varying 


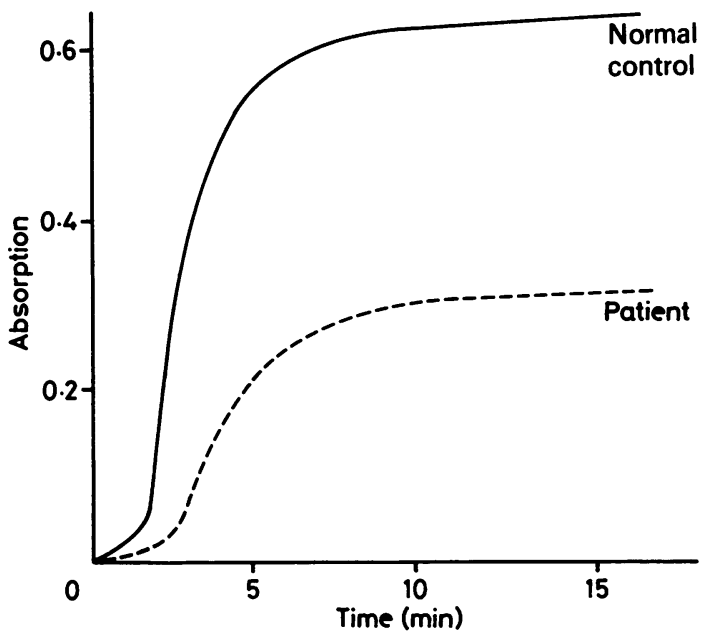

Fig 3 Fibrin polymerisation measured by change in optical density of fibrin clot against time by method of Belitser et al. ${ }^{6}$ Fibrin polymerisation is impaired compared with that of normal control.

molecular weights. This pattern of A-alpha chain degradation is consistent with $\mathrm{C}$-terminal A-alpha chain degradation by plasmin or trypsin-like enzymes. ${ }^{5}$

\section{POLYMERISATION OF PURIFIED FIBRIN} MONOMERS

Fibrinogen that had been isolated from citrated plasma was clotted with thrombin, dissolved in dilute acetic acid, and polymerised by increasing the $\mathrm{pH}$ by adding phosphate buffer, as described by Belitser et al. ${ }^{6}$

Fig 3 shows that polymerisation of the patient's fibrin was impaired compared with that of a control. This was almost certainly caused by the extensive Cterminal degradation and explains the prolongation of the thrombin time.

Coagulation tests and fibrinogen studies were repeated six months following discharge and yielded normal results.

\section{Discussion}

This patient had acquired dysfibrinogenaemia occurring during acute pancreatitis secondary to stones in the pancreatic duct. The dysfibrinogenaemia was not associated with a bleeding problem. Initially it was suspected that DIC was the sole cause of the abnormal coagulation findings, particularly as the serum fibrinogen degradation product value was grossly increased. It has been suggested that DIC can be precipitated in pancreatitis through the action of circulating pancreatic proteases, especially trypsin, on the coagula- tion factors, so triggering the clotting cascade. ${ }^{7}$ The finding of a raised D-dimer concentration (a marker of circulating products from the degradation of crosslinked fibrin) and the slight reduction in the ratio of fibrinopeptide A to B release from the fibrinogen in plasma (suggesting the presence of fibrin monomer) indicates that DIC was probably present. The persistently normal platelet count and prothrombin time would suggest that the DIC was only mild.

There were two main observations which indicated that DIC may not have been the sole cause of the coagulation abnormalities, prompting investigation for the presence of a dysfibrinogenaemia. Firstly, although a prolonged thrombin time can occur in DIC as a consequence of hypofibrinogenaemia or inhibition of the thrombin-fibrinogen interaction by circulating fibrinogen degradation products, ${ }^{8}$ it would be unusual for this to occur in the absence of abnormalities of other clotting tests. Secondly, a close relation exists between serum fibrinogen degradation product and D-dimer concentrations in DIC. ${ }^{9}$ In this case the serum fibrinogen degradation product value was disproportionately higher than would have been expected for the observed D-dimer concentration. Although degradation products resulting from the DIC would have contributed towards the increased value of fibrinogen degradation product level, it was thought possible that the very high value obtained was, in part, a spurious finding due to the detection of functionally abnormal fibrin which had remained in the serum following the in vitro preparation of the test sample.

Further studies showed a loss of peptides from the C-terminus end of the A-alpha chains of the fibrinogen. We suggest that this occurred as a consequence of the action of circulating pancreatic proteases either directly on the fibrinogen or indirectly via activation of plasminogen to plasmin. This proteolytic cleavage was presumably the cause of the reduced polymerisation of purified fibrin observed in vitro and explains the prolonged thrombin time. The close temporal relation between the increased amylase activities and the prolonged thrombin time which persisted for three weeks following admission would support a case for implicating pancreatic proteases as the cause of the dysfibrinogenaemia. It would be unlikely for the degree of fibrinogenolysis observed to have been a consequence of fibrinolytic activation secondary to the coexisting DIC.

As far as we are aware, an acquired abnormality of fibrinogen of the severity described here occurring in association with acute pancreatitis has not previously been reported. The only reported cases of acquired dysfibrinogenaemia have occurred in association with liver disorders. ${ }^{10-12}$ The defect in these cases seems to be due to an abnormality in fibrin monomer polymerisa- 
tion. It has been suggested that this arises because of the development of abnormalities of the carbohydrate moieties in the fibrinogen molecule as a result of liver dysfunction ${ }^{13}$ but increased proteolytic degradation of fibrinogen may also be a factor. ${ }^{14}$ Acquired dysfibrinogenaemia may be an under-recognised phenomenon in acute pancreatitis and could lead to misinterpretation of coagulation test abnormalities resulting in the administration of inappropriate treatment.

We thank Mrs E Thompson for performing the fibrinogen studies.

\section{References}

1 Lasson A, Ohlsson K. Consumptive coagulopathy, fibrinolysis and protease-antiprotease interactions during acute human pancreatitis. Thromb Res 1986;41:167-83.

2 Southan C, Thompson E, Lane DA. Direct analysis of plasma fibrinogen-derived fibrinopeptides by high performance liquid chromatography. Thromb Res 1986;43:195-204.

3 Jacobson E, Kierulf P. A modified beta-alanine precipitation procedure to prepare fibrinogen free of anti-thrombin 111 and plasminogen. Thromb Res 1973;3:145-59.

4 Weber K, Osborn M. The reliability of molecular weight determinations by dodecyl sulphate-polyacrylamide gel electrophoresis. J Biol Chem 1969;244:4406-12.
5 Gaffney PJ. Subunit relationships between fibrinogen and fibrin degradation products. Thromb Res 1973;2:201-12.

6 Belitser VA, Varetskaja TV, Malneva GV. Fibrinogen-fibrin interaction. Biochim Biophys Acta 1968;154:367-75.

7 Lasson A, Ohlsson K. On the potential role of trypsin and trypsin inhibitors in acute pancreatitis. Adv Exp Med Biol 1984;167:477-87.

8 Marder VJ, Shulman NR. High molecular weight derivatives of human fibrinogen produced by plasmin. II. Mechanism of their anticoagulant activity. J Biol Chem 1969;244:2120-4.

9 Wilde JT, Kitchen S, Greaves M, Preston FE. A comparison of plasma D-dimer and serum fibrinogen/fibrin degradation product levels in the investigation of hypercoagulable states. In: Lowe GDO, ed. Fibrinogen biochemistry, physiology and clinical relevance: workshop proceedings. Amsterdam: Excerpta Medica, 1987.

10 Samama M, Soria J, Soria C. Congenital and acquired dysfibrinogenaemia. In: Recent advances in blood coagulation. Edinburgh: Churchill Livingstone, 1976:313-35.

11 Green G, Thomson JM, Dymock IW, Poller L. Abnormal fibrin polymerisation in liver disease. Br J Haematol 1976;34:427-39.

12 Lane DA, Scully ME, Thomas DP, Kakkar VV, Woolf IL, Williams R. Acquired dysfibrinogenaemia in acute and chronic liver disease. Br J Haematol 1977;35:301-7.

13 Martinez J, Palascak JE, Kwasniak D. Abnormal sialic acid content of the dysfibrinogenaemia associated with liver disease $J$ Clin Invest 1978;61:535-8.

14 Soria J, Soria C, Ryckewaert JJ, Samama M, Thomson JM, Poller L. Study of acquired dysfibrinogenaemia in liver disease. Thromb Res 1980;19:29-41.

Requests for reprints to: Dr J T Wilde, Department of Haematology, Royal Hallamshire Hospital, Glossop Road, Sheffield S10 2JF, England. 